\title{
Wearing N95, Surgical, and Cloth Face Masks Compromises the Perception of Emotion
}

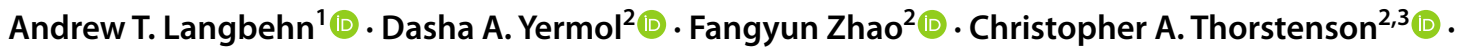 \\ Paula M. Niedenthal ${ }^{2} \mathbb{D}$
}

Received: 10 May 2021 / Accepted: 2 December 2021/Published online: 26 January 2022

(c) The Society for Affective Science 2022

\begin{abstract}
According to the familiar axiom, the eyes are the window to the soul. However, wearing masks to prevent the spread of viruses such as COVID-19 involves obscuring a large portion of the face. Do the eyes carry sufficient information to allow for the accurate perception of emotions in dynamic expressions obscured by masks? What about the perception of the meanings of specific smiles? We addressed these questions in two studies. In the first, participants saw dynamic expressions of happiness, disgust, anger, and surprise that were covered by N95, surgical, or cloth masks or were uncovered and rated the extent to which the expressions conveyed each of the same four emotions. Across conditions, participants perceived significantly lower levels of the expressed (target) emotion in masked faces, and this was particularly true for expressions composed of more facial action in the lower part of the face. Higher levels of other (non-target) emotions were also perceived in masked expressions. In the second study, participants rated the extent to which three categories of smiles (reward, affiliation, and dominance) conveyed positive feelings, reassurance, and superiority, respectively. Masked smiles communicated less of the target signal than unmasked smiles, but not more of other possible signals. The present work extends recent studies of the effects of masked faces on the perception of emotion in its novel use of dynamic facial expressions (as opposed to still images) and the investigation of different types of smiles.
\end{abstract}

Keywords Facial expression $\cdot$ Facial Perceptions $\cdot$ Nonverbal behavior $\cdot$ Smiles $\cdot$ COVID-19

\section{Introduction}

For most people, life during the COVID-19 pandemic may have felt flat and lacking in the full range and complexity of social stimulation. Indeed, people typically see each other's faces signaling states as varied as disgust, awe, effort, frustration, and attraction, but during

Handling Editor: Rachael Jack

Paula M. Niedenthal

niedenthal@wisc.edu

1 Department of Psychology, University of TennesseeKnoxville, Knoxville, TN, USA

2 Department of Psychology, University of Wisconsin-Madison, Madison, WI, USA

3 Wisconsin Institute for Discovery, University of Wisconsin-Madison, Madison, WI, USA the pandemic, we saw fewer faces, and the faces we did see were partly covered by masks. The use of masks, of course, will continue as policy makers respond to mutations of the virus such as the Delta variant, and to future pandemics. While the health benefits are undeniable, a potential adverse effect of the use of face masks is that it reduces the amount of information conveyed by the face.

The overall loss of visual information is significant. Human faces are composed of 43 individual muscles that possess the unique property of being connected to skin and other muscles, but not to bone. The muscles can contract in a variety of combinations, producing the temporary changes in the superficial geometry of the face that we know as facial expressions (Cattaneo \& Pavesi, 2014; Ekman et al., 1971). Facial expressions are nuanced social signals. They efficiently communicate behavioral intentions, attitudes, as well as emotional states (Crivelli \& Fridlund, 2014; Jack \& Schyns, 2015; Kret et al., 2020), and a perceiver's ability to 
accurately extract meaning from facial expressions is important for successful social interaction (Hastings et al., 2008; Marsh \& Blair, 2008; Niedenthal \& Brauer, 2011).

Is a person's ability to accurately process the meaning of facial expression compromised when the perceived face is covered by a mask? The question must be answered empirically because the possibility that people's internal states and momentary intentions can be perceived from the eyes alone is a plausible alternative hypothesis (Baron-Cohen et al., 2001; Schmidtmann et al., 2020). The current work investigated the perception of emotion in dynamic human facial expressions that were fully visible versus faces covered by the most common face masks in use during the COVID-19 pandemic: N95, surgical, and cloth. ${ }^{1}$ In Study 1, we explored the effects of mask wearing on expressions of some so-called basic emotions (Keltner et al., 2003), and in Study 2 we expanded our investigation into the effects of mask wearing on more nuanced variations of the human smile (Rychlowska et al., 2017).

In theory, perceiving emotions in facial expressions relies on at least three processes that can be hampered by masks (Wood et al., 2016). First, visual information from faces can be matched to mental representations of expressions that have been encountered and labeled in the past. For example, for most people, downturned lips on a face have throughout their lives been repeatedly associated with the label "sad" or with behavioral indicators of sadness, such as crying. Face masks occlude some of the visual information used in this matching process. Second, emotion recognition can also rely on proprioceptive feedback from the perceiver's face; the feelings of mimicking the expression on someone else's face can provide essential input into the process of interpreting the expression (Wood et al., 2016). Because masks reduce visual information conveyed by the face, they can disrupt the production of corresponding facial mimicry in a perceiver. Finally, people rely on contextual information to interpret emotion expressions (Hassin et al., 2013). Face masks, because they make expressions more ambiguous, may increase the use of pre-existing stereotypes and expectations about emotions that fit the context, which may or may not result in an accurate interpretation of the expression (Maringer et al., 2011).

\footnotetext{
${ }^{1}$ N95 masks are particulate filtering face masks in the USA, certified by the National Institute of Occupational Safety and Health (NIOSH) to filter at least $95 \%$ of airborne particles (see Fig. 1).
}

For example, when displaying randomly selected portions of the face (i.e., the "bubbles" technique), it has been shown that the mouth is a primary diagnostic area for the accurate perception of the six so-called basic emotions as well as pain and neutral expressions (Blais et al., 2012). However, participants were still able to accurately categorize facial expressions from only the eyes, but with less frequency (Saumure et al., 2018). Further, categorizations of target emotions are also impaired when 'freezing' the eyes, and to a greater extent the mouth, during dynamically displayed stimuli (Nusseck et al., 2008). These findings suggest that blocking signals from the lower portion of the face impairs perceptions of emotion. However, these methods do not precisely match how we perceive expressions on masked faces. A plausible alternative interpretation of these findings is that the unnatural techniques used to mask portions of the face reduce perceiver's accuracy beyond that due to blocking signals from the face.

Any adverse effect of information occlusion caused by mask-wearing may also depend upon the degree to which information from a specific emotion expression-its signal-is largely conveyed on the lower or upper part of the face (Calvo \& Nummenmaa, 2008; Smith et al., 2005). One method for describing the facial muscle actions that comprise emotion expressions is the Facial Action Coding System (FACS; Ekman et al., 2002). In this system, expressions of happiness (also termed reward smiles in the present report) are comprised of the lip corner puller (called action unit [AU] 12) and the cheek raiser, which causes crow's feet (AU 6; see Fig. 2 for the action units for each of the four emotions examined here). The disgust expression is composed of the nose wrinkler (AU 9), the lip corner depressor (AU 15), and the lower lip depressor (AU 16). In both expressions, a large portion of their signal is thus located in the lower part of the face. In contrast, the anger expression is composed of the actions of three muscles on the upper part of the face (AUs 4, 5, and 7 , brow lowerer, upper eyelid raiser, eyelid tightener, respectively) and one muscle on the lower part of the face, the lip tightener (AU 23). Surprise is also composed of three facial action units in the upper face (AUs 1, 2, and 5; inner brow raiser, outer brow raiser, upper eyelid raiser) and one action, jaw drop (AU 26), in the lower face. The perception of expressions with predominantly lower face action units (happiness and disgust) may be impacted by face masks to a greater degree than those utilizing predominantly upper face musculature (anger and surprise).

The reasoning thus far suggests that masks reduce the amount of a target emotion perceived for some, if not all, facial expressions: a smile looks less happy, and a disgust expression looks less disgusted. However, 
there is also reason to expect that occluding the face can facilitate the perception of other, non-target emotions as well. For example, faces covered by a niqab or a black rectangle elicit perceptions of smiles and expressions of shame as conveying more non-target negative emotions compared with fully visible faces (Fischer et al., 2012). Further, when mimicry of another person's smile was inhibited, people were more likely to interpret the smile in terms of contextual information, leading to less accuracy in perceiving the genuineness of the smile (Maringer et al., 2011).

Several recent studies have investigated the effects of face masks on the interpretation of facial expressions. Faces expressing happiness, sadness, anger, and disgust while covered with masks were misclassified as conveying alternative emotional states more often than their fully visible counterparts (Carbon, 2020). This difference was not observed for expressions of fear or neutral emotion. Marini and colleagues (Marini et al., 2021) found that opaque face masks reduced the accuracy of emotion perception for happiness, fear, and surprised expressions. However, when covered by a transparent mask, emotion recognition was not significantly different from fully visible faces. Children also display a marked decrease in their ability to recognize masked expressions of emotion. Carbon and Serrano (2021) found that children, 9-10 years old, were less accurate at recognizing expressions of disgust, fear, happiness, and sadness when the expressions were covered by a face mask. However, they find a surprising increase in accuracy for anger and neutral expressions.

Taken together, these research findings indicate that masked expressions communicate less of the intended emotion especially if the expression involves significant lower face action. While such findings are supportive, all prior studies employed static photographs as facial expression stimuli, while it has been repeatedly demonstrated that much of the information from an emotion expression is conveyed through its dynamic properties, that is, the timing of the contraction of participating muscles (i.e., facial motion; Ambadar et al., 2005; Back et al., 2009; Kamachi et al., 2013). The present two studies therefore employed dynamic facial expression videos rather than static images. Each of the three studies discussed above utilized a forced-choice paradigm. This design feature necessarily limited possible responses to the presented expressions. In contrast, the present study utilized a continuous measure of the extent to which each face expressed each of four emotions, which allowed respondents to report more nuanced perceptions of the expressions.

In Study 1, we investigated the perception of dynamic expressions of happiness, disgust, anger, and surprise that were fully visible, covered by one of three types of masks
(N95, surgical, or cloth), or occluded by a white rectangle which was used to control for attitudes towards mask wearing. In Study 2, we investigated the perception of different types of smiles, including reward, affiliation, and dominance smiles as described by Martin and colleagues (Martin et al., 2017, and see below). Research on masks and emotion perception has yet to investigate the impact of masks on more nuanced social signals such as the three types of smiles. Additionally, the use of a mask to protect oneself from the spread of COVID-19 has become politicized in the United States (Pew Research Center, 2020), so we examined the possible moderating role of political attitudes on the effects of masks on the perception of dynamic facial expressions in both studies. The moderating role of gender of the participant-perceiver was also examined since a recent metaanalysis confirmed that women are on average more accurate than men in recognizing facial expressions (Thompson \& Voyer, 2014).

\section{Study 1: Effects of Masks on Perceiving Happiness, Disgust, Anger, and Surprise}

\section{Methods}

\section{Participants}

A total of 252 MTurk workers were recruited to participate in a "survey about facial expressions." Those with lower than a 95\% HITT rate were excluded. Of the total, 162 MTurk workers ranging in age from 20 to $73(M=36.46, S D=10.85)$ provided informed consent, completed the study, passed the attention checks, and were included in the analyses. The final sample included 89 males and was composed of individuals of whom 116 were White, 32 African American, 9 Asian, and 5 Native American, Native Alaskan, or Other. Participants received $\$ 5$ in compensation for completing the survey, which took them on average $40 \mathrm{~min}$. Compensation was based on past research using Mturk and balanced thoughtful participation while maximizing sample size. Sample size was preregistered and is commensurate with previous research (Carbon, 2020) that utilized a sample of $N=36$ for a single mask design.

\section{Materials}

\section{Masks}

We collected royalty-free images of masks through an online image search. Three masks were chosen 


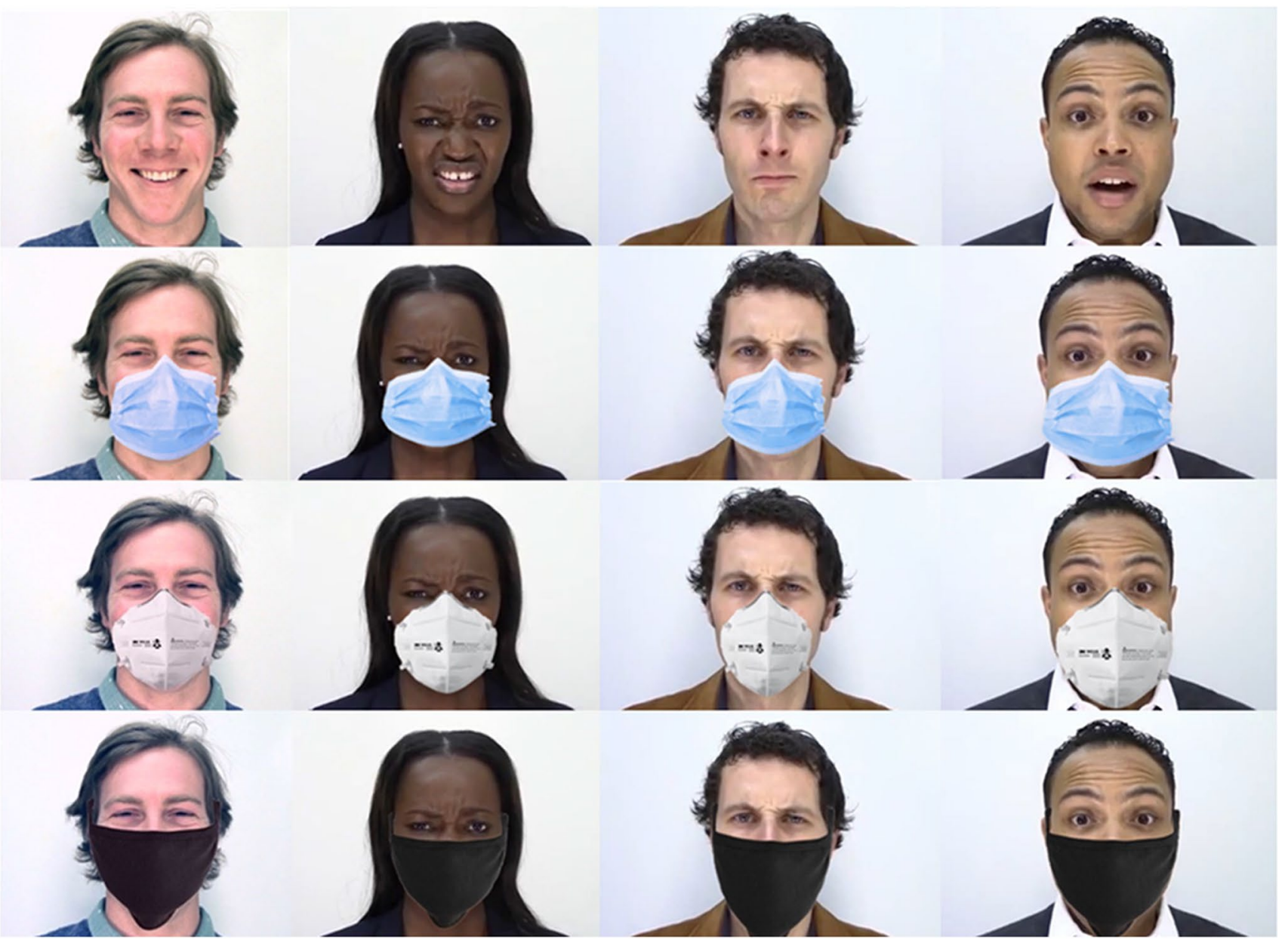

Fig. 1 Dynamic expression stimuli for study 1. The top panel shows still images taken from faces dynamically expressing, from left to right, happiness, disgust, anger, and surprise. The lower panels show

to represent those most commonly worn during the COVID-19 pandemic including the N95, a blue surgical, and a black cloth mask (Fig. 1). The N95 and blue surgical mask were collected from CleanPNG.com and the black cloth mask was found through a Google Image search for "royalty free black cloth mask images." We created the white rectangle using Microsoft 3D Paint and included it as a neutral facial occlusion to control for attitudes toward mask wearing.

\section{Emotion Expression Videos}

Expression videos were selected from a large database of short, 1 to $3 \mathrm{~s}$, pre-recorded facial expression videos (Rychlowska et al., 2017). The final stimulus set included videos of 14 actors ( 3 Black females, 4 White females, 4 Black males, 3 White males) expressing smiles, anger, images from the same faces covered by a surgical mask, N95 mask, and black cloth mask

disgust, and surprise (42 total videos). Note that not all actors expressed every one of the four emotion expressions. As discussed above, we used the expressions of anger and surprise, because in their prototypic form they involve more facial actions in the upper part of the face, and happiness and disgust because they involve more facial action in the lower part of the face (Fig. 2). While the videos have previously been normed, FACS coding of them was not performed for the present study. The fully visible conditions of the study served as a manipulation check on the level of target emotion communicated by each expression for the present samples.

\section{Dynamic Emotion Expression Stimuli}

Video stimuli were created using Davinci Resolve 16, a video editing application. We selected a single point on each actor's face and used point-tracking to track actors' movements during the expressions. The location of the point tracking varied 


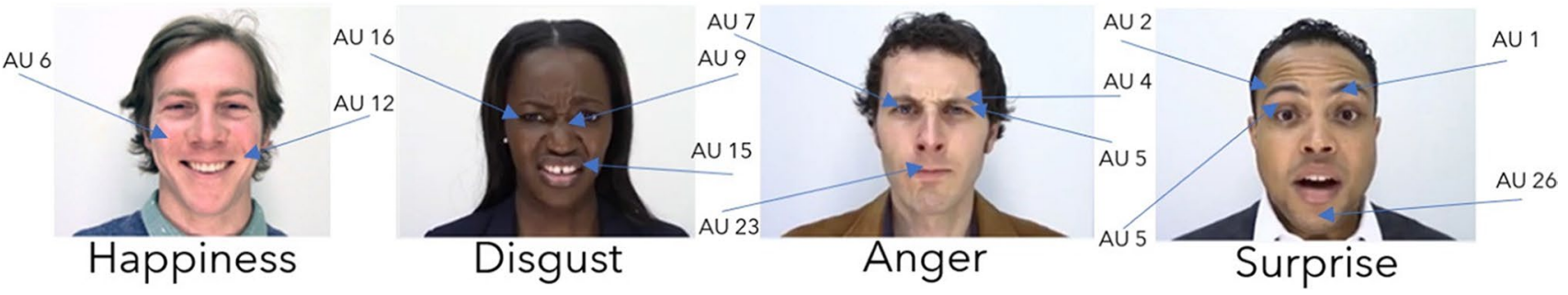

Fig. 2 FACS coding of happiness, disgust, anger, and surprise expressions. Happiness is characterized by AUs 6, the cheek raiser, and 12 the lip corner puller. Disgust expressions are predominately comprised of AUs 9 the nose wrinkle, 15 the lip corner puller, and 16 the lower lip depressor. Anger expressions utilize AUs 4 the brow

due to differences in individual face morphology, and luminance and contrast of the video. The three masks and white rectangle were overlayed on each expression video, which yielded, together with the unmasked videos, 210 unique dynamic expression stimuli. The background of the masks was removed, and the masks and white rectangle were sized to fit the actor's face. The white rectangle was sized to conceal the approximate amount of the actor's face as the three masks. The top, middle of each mask, and the white rectangle were placed on the bridge of the nose, so they extended approximately 1-inch below the actor's eyes (Fig. 3). The masks and white rectangle overlay were then matched to the movement of the actor which ensured that the mask and white rectangle occluded the actor's face over the duration of the video to the same degree (Video stimuli can be found at https://osf.io/ rbhxd/?view_only=1f01c0205c884f54b7472fb3a617c15b).

\section{Survey}

Data were collected via a survey hosted on the Qualtrics online survey platform. Participants completed the study in a location and on a device of their choosing. Video

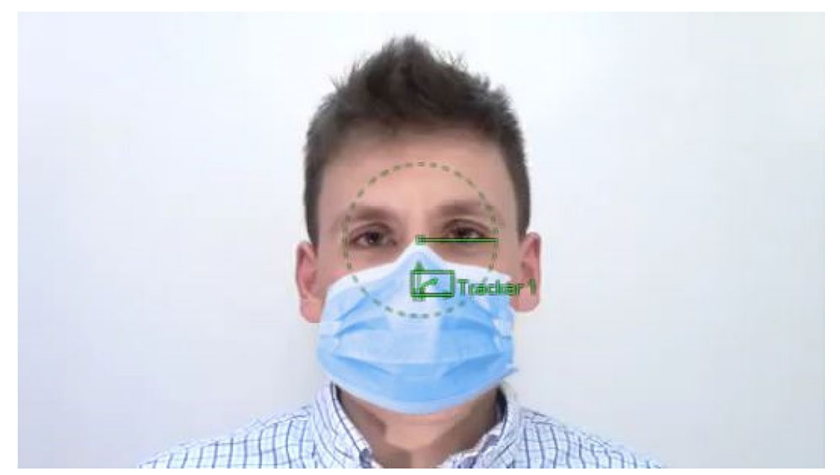

Fig. 3 Mask location for dynamic emotion expression stimuli. The green line in the center of the image represents the tracked point of the actor's face and movement throughout the video lowerer, 5 the upper eyelid raiser, 7 the eyelid tightner, and 23 the tightner. Surprise expressions are typically comprised of AUs 1, 2, 5, and 26 the inner brow raiser, outer brow raiser, upper eyelid raiser, and jaw dropper respectively

stimuli were embedded in the Qualtrics survey. Given the variety of possible viewing modalities, the viewing angle and distance, central visual location, size of the video, and other variables are unknown and a limitation of the present research. Video stimuli of facial expressions (happy, disgust, anger, surprise) for each actor were crossed with rating scale (happiness, disgust, anger, surprise) and face condition (mask or visible) resulting in 840 dynamic expression stimuli and rating scale pairings. The expression-rating scale pairings were first divided into four surveys according to mask type (N95, surgical, cloth, white rectangle), with all four surveys including visible face videos. Therefore, each survey contained 332 pairings which we further divided into two versions containing half of the video stimuli resulting in 168 trials per survey. The surveys were constructed such that participants did not see a given actor expressing the same emotion with and without a mask. The two versions were equated for the race (African American, White) and gender (male, female) of the actors and included four attention check questions. Participants rated the emotion expressed in each video by responding to the prompt, "How much (happiness/disgust/anger/surprise) is this person expressing?", on a 100 -point scale $(0=$ none to $100=$ a great deal).

Demographic questions were included at the end of the survey. Participants reported their age, gender, and race, along with their political ideology on a 7-point scale $(1=$ very conservative to, $7=$ very liberal $)$, and geographical location. Geographical location was assessed on two dimensions, rural versus urban, and state and city of residence. The researchers also included two validity checks at the end of the survey, "Did you watch each video before answering the question?" and "Did you experience any problems with the videos or survey?".

\section{Procedure}

In Study 1, 162 workers on Mechanical Turk (MTurk; Amazon's on-demand micro-task platform) viewed 168 videos of 
14 different actors displaying happiness, disgust, anger, and surprise (factor "expression"). The actors' faces displayed neutral emotion at the beginning of the videos and then changed to express a particular emotion. In half of the trials, the face was fully visible, whereas on the remaining trials, the face was masked (factor "face presentation," see Fig. 1). The facecovering was an N95 mask, a blue surgical mask, a black cloth mask, or a white rectangle (factor "mask type"). Mask type varied between participants such that participants only saw one type of mask or the white rectangle throughout the study (final $N$ 's were approximately 40 per mask type condition).

The four emotion expressions were seen an equal number of times on visible and masked trials. Expression videos were randomly presented four times, and participants rated the extent to which the actor was expressing each emotion: once for happiness, once for disgust, once for anger, and once for surprise. Thus, for every emotion expression on both fully visible and masked faces, we acquired ratings of the target emotion as well as the three non-target emotions (factor "rating scale").

\section{Results}

Analyses revealed the predicted two-way interaction between face presentation and rating scale, $F(1,158)=277.52$, $p<0.001, \eta_{\mathrm{p}}{ }^{2}=0.637$. Specific tests reported in Fig. 4 indicate that, as expected, for all expressions, the target emotion was perceived as less present in masked versus visible faces. That is, happy, disgust, angry, and surprised expressions were perceived as less happy, disgusted, angry, and surprised, respectively, when masked compared with fully visible.

Participants also perceived more of the non-target emotions in the masked faces in almost all cases as reported in the caption. Specifically, happy expressions, when masked, were rated as expressing more anger $t(161)=6.77$, $p<0.001, d=0.53$, and disgust $t(161)=7.70, p<0.001$, $d=0.60$, but not more surprise, $t(161)=0.79, p=0.43$. When masked, disgust expressions were rated as expressing more happiness, $t(161)=6.46, p<0.001, d=0.51$ anger $t(161)=6.53, p<0.001, d=0.51$, and surprise $t(161)=2.90$, $p=0.004, d=0.23$. Anger expressions were seen as expressing more happiness, $t(161)=5.28, p<0.001, d=0.41$, disgust, $t(161)=2.69, p=0.008, d=0.21$, and surprise $t(161)=4.21, p<0.001, d=0.33$, when masked. Analyses revealed a slightly different pattern for surprised expressions. Surprise expressions were rated as expressing less surprise when masked $(M=77.1, S E=1.19)$, compared to when fully visible $(M=83.2 .1, S E=1.09), b=6.07$, $s e=1.04, t(161)=5.84, p<0.001, d=0.46$. However, fully visible surprised faces $(M=30.1, S E=1.50)$ were rated as expressing more of the non-target emotions than masked faces $(M=24.9, S E=1.81) b=5.25$, $s e=0.73, t(161)=7.21$, $p<0.001, d=0.57$. Decomposing the non-target ratings for surprised expressions we found that masked surprised faces were rated as more angry $b=4.16, s e=0.89, t(161)=4.70$, $p<0.001, d=0.37$ and more disgusted $b=5.68$, se $=0.95$, $t(161)=5.98, p<0.001, d=0.47$. However, masked surprise
Fig. 4 Emotion ratings of visible and masked faces. The graphs illustrate levels of emotion perceived in videos of visible and masked faces dynamically expressing happiness, anger, disgust, and surprise. Separate t-tests comparing the perception of emotion in each expression indicate that participants perceived significantly less of the target emotion $(p s<.001)$, and significantly more of most non-target emotions $(p s<.008)$ in masked compared to visible faces. Two exceptions of note were that surprise was not perceived as more present in masked happy expressions $(p=.43)$ and happiness was perceived as significantly less present in masked surprise expressions $(p<.001)$. $* p<.05, * * p<.01, * * * p<.001$
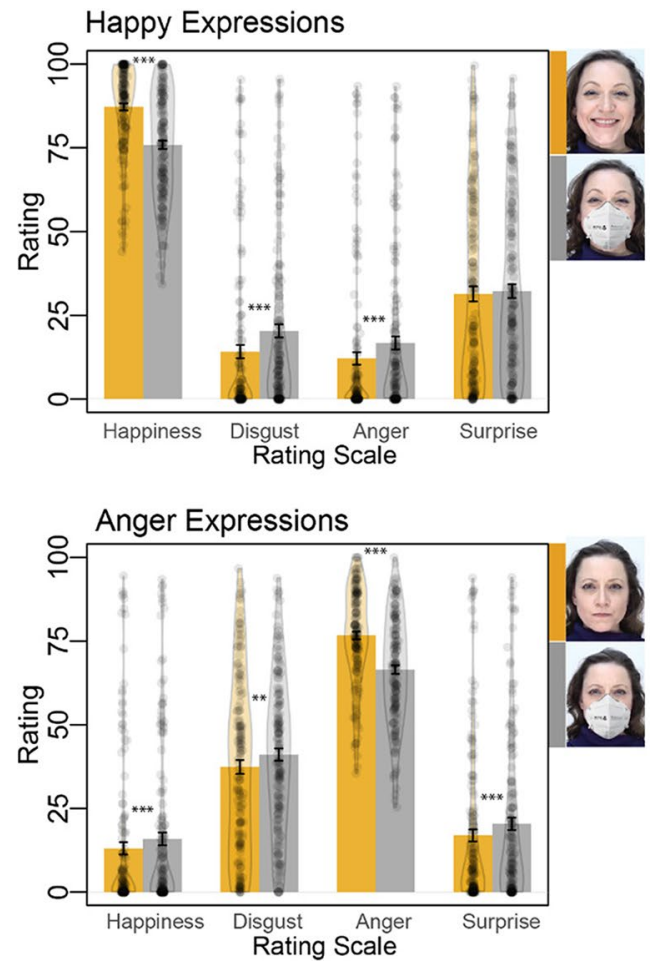

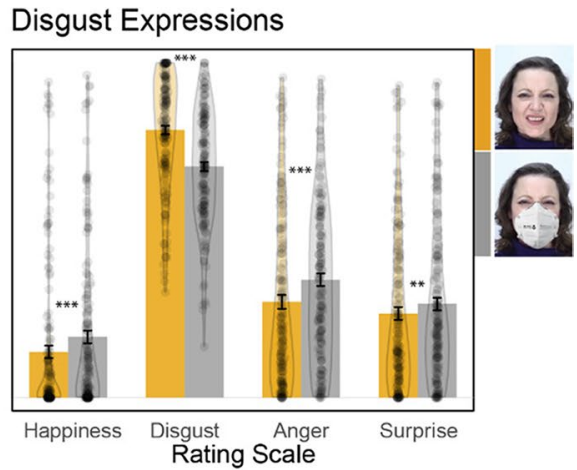

Surprise Expressions

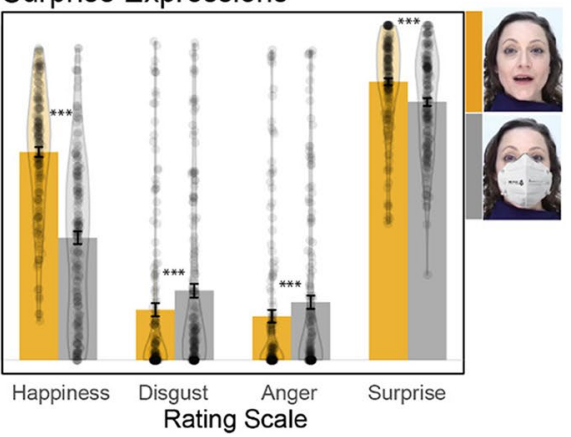


faces were also rated as less happy, $b=25.60$, $s e=1.68$, $t(161)=15.27, p<0.001, d=1.97$. Taken together, our results indicate that masked expressions communicate less of the target emotion and more of the non-target emotions with the exception of surprised and happy faces which were rated as expressing more happiness and surprise respectively when fully visible as compared to when masked. This is likely, in part, due to our surprised expression stimuli expressing happy as opposed to fearful surprise.

The perception of facial expressions occluded by masks varied by whether the expression prototypically carries more of its signal on the upper versus lower part of the face. There was a significant two-way interaction between face presentation (visible, masked) and facial action predominance (upper, lower face), $F(1,158)=7.17, p=0.008, \eta_{\mathrm{p}}{ }^{2}=0.043$, indicating that perceiving the target emotion in happy and disgust expressions (with more signal in the lower part of the face) was compromised to a greater degree than perceiving the target emotion in angry and surprised expressions (with more signal in the upper part of the face).

Political attitudes did not uniformly moderate any of the findings, nor were they moderated by gender of participant or the race of the actor (see S1 Appendix, Appendix 1). Further statistical analyses for Study 1 are also described in S1 Appendix, Appendix 1.

\section{Study 2: Effects of Masks on Perceiving Reward, Affiliation, and Dominance Smiles}

The first study examined prototypical expressions of some of the so-called basic emotions on masked versus fully visible faces, but many if not most expressions seen "in the wild" are more nuanced. Imagine stopping to pick up after your dog on a sidewalk of a bustling urban center. A person wearing a face mask passes you, makes eye contact, and appears to express something. What is it? The person seems to be smiling, but are they smiling with disdain or gratitude? Are they commiserating because they are also a dog owner? Your classification of the smile will determine your response.

Smile expressions typically involve the activation of the muscle that causes the corners of the lips to rise (i.e., AU 12 in the FACS), but there is variability in the degree to which other actions are present and even the extent to which a participating muscle is activated bilaterally. In fact, recent research supports the existence of more than one type of smile, and while all types are honest social signals that have predictable effects on a perceiver's physiology and behavior (e.g., Martin et al., 2017, 2021), they do not all communicate happiness. A social-functional account of the apparent heterogeneity of human smiles holds that different forms of smiles are deployed to accomplish distinct social tasks (Martin et al., 2017; Niedenthal et al., 2010). Reward smiles reinforce desired behavior in the self and others (i.e., they shape behavior), affiliation smiles communicate that the smiler is not a threat and is open to safe interaction (i.e., they invite affiliation), and dominance smiles are used to convey criticism and social status (i.e., they are used to negotiate social hierarchies; Martin et al., 2017). Recent studies have provided quantitative descriptions of the forms of these three social-functional smiles, which are shown in Fig. 5 (e.g., Niedenthal \& Brauer, 2011).

The reward smile, which most people view as expressing happy feelings (and was employed in Study 1), is symmetrical and usually involves open lips, whereas the dominance smile is viewed as expressing far less happiness, is quite asymmetrical, and sometimes contains facial actions seen in contempt, including the nose wrinkler (AU 9) and the lip raiser (AU 10). English language speakers use the terms "smirk" or "smug smile" to describe this combination of features. The affiliation smile contains unique actions involving the dimpler (AU 14) and the lip presser (AU 24), which pull the upper lip across the teeth making for an expression that communicates appeasement and approachability. Returning to the previous example, a passerby could conceivably communicate reassurance or disdain as they observe you picking up after your dog in
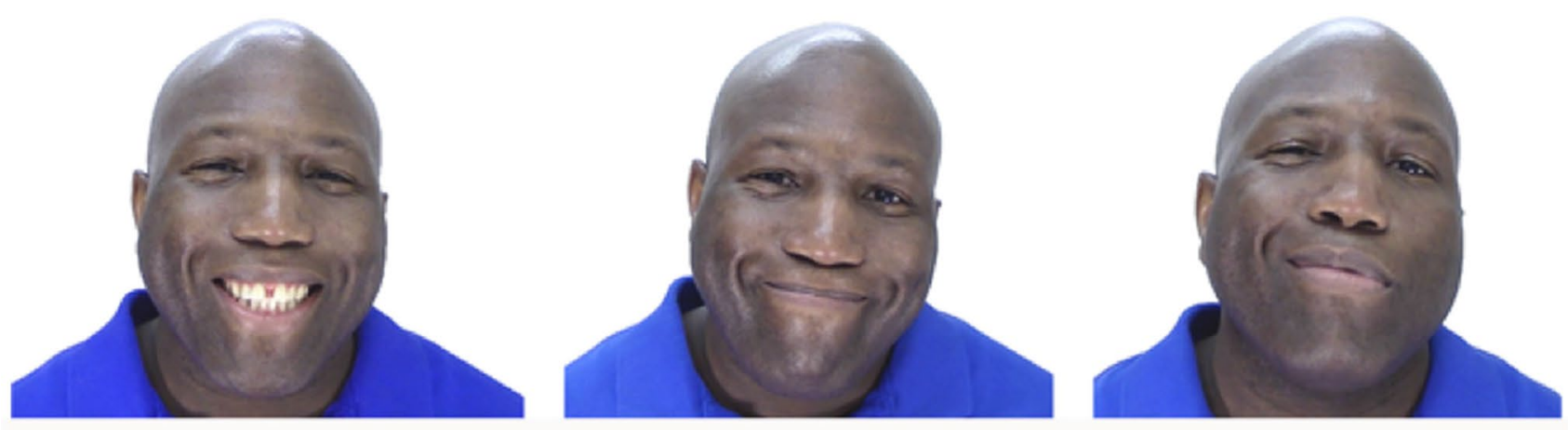

Fig. 5 Examples of smile stimuli. Reward (left), affiliation (center), and dominance (right; Martin et al., 2021) 
a dense urban area, or they might even reward you for fulfilling what many consider a civic duty. The morphological distinction among the smiles is largely evident on the lower part of the face. So, detecting the meaning of a particular smile could be difficult when the person is wearing a mask.

In Study 2, participants saw videos of reward, affiliation, and dominance smiles in both fully visible and masked presentations. Because the major findings from Study 1 did not interact with mask type, only the N95 mask was used in this study. Participants rated all smiles in terms of the extent to which each conveyed positive feelings, reassurance, and superiority, which we selected to serve as labels for the signals communicated by reward, affiliation, and dominance smiles, respectively. Note that while we used labels derived from our scientific taxonomy of the smile, these labels might not be used by laypeople, who, on the other hand, typically have shared knowledge about expressions of the so-called basic emotions and their labels.

\section{Methods}

\section{Participants}

A total of 93 MTurk workers were recruited to participate in a "survey about facial expressions." Those with lower than a 95\% HITT rate were excluded. Of the total, 60 MTurk workers ranging in age from 23 to $68(M=35.25, S D=9.01)$ provided informed consent, completed the study, passed the attention checks, and were included in the analyses. The final sample included 36 male participants, 41 were White, 17 African American, and 2 Asian or Other. Participants received $\$ 4$ in compensation for completing the study that took on average $32 \mathrm{~min}$. Compensation was based on past research using MTurk and to encourage thoughtful participation while maximizing sample size. Sample size was preregistered and is commensurate with previous research (Carbon, 2020) that utilized a sample of $N=36$ with a six groups (emotions) design.

\section{Materials}

\section{Dynamic Expression Stimuli}

Smile expression videos were selected from the same database of pre-recorded facial expression videos as Study 1 (Martin et al. 2021; Rychlowska et al., 2017). The stimulus set included videos of 14 actors ( 3 black females, 4 white females, 4 black males, 3 white males) expressing reward, affiliation, and dominance smiles (Fig. 6) totaling 34 stimuli. Note that not every actor expressed all three smiles. Because there were no important interactions with mask type found in Study 1, only the N95 mask was applied to the smile videos, again with DaVinci Resolve 16 and following the same steps outlined in Study 1.

Video stimuli of smile expressions were crossed with rating scale (positive feeling, reassurance, superiority) and face condition (mask or visible) resulting in 204 dynamic expression stimuli and rating scale pairings.

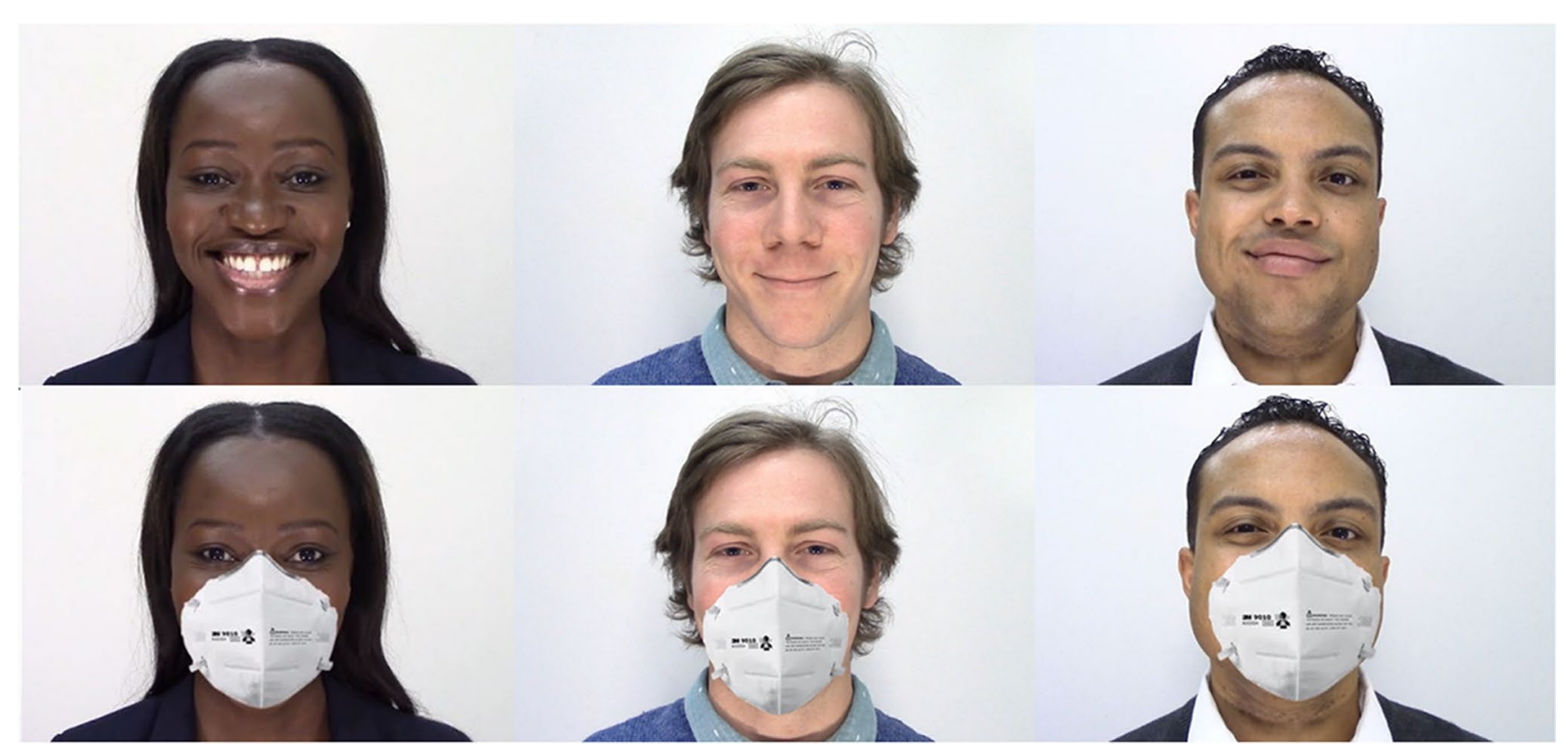

Fig. 6 Masked and visible dynamic smile stimuli. The top panel shows images taken from dynamic videos of, from left to right, reward, affiliation, and dominance smiles. The lower panel show images from the same faces covered by the N95 mask 


\section{Survey}

Data were collected via a survey hosted again on the Qualtrics online survey platform. Expression stimuli were divided into two versions of the survey to ensure participants did not see an actor expressing the same type of smile both fully visible and masked. Participants were randomly assigned to be in one of the two versions of the survey and stimuli were randomly presented within each version. The two versions were equated for the race and gender of the actors. Each version of the survey contained 102 dynamic expression stimuli which participants rated on the same 100-point scale, "How much (positive feeling/reassurance/superiority) is this person expressing?" Study 2 included three attention check questions and participants completed the same demographics questionnaire and validity check questions as Study 1.

\section{Results}

We started by confirming that smiles of reward, affiliation, and dominance, when fully visible, were perceived as communicating the target signals we had labeled as positive feelings (reward smiles), reassurance (affiliation smiles) and superiority (dominance smiles) see Fig. 7. Reward smiles were seen as signaling significantly more positive feelings $(M=72.5, S E=1.99)$ than reassurance and superiority $(M=59.1, S E=2.43), t(59)=5.25$, $p<0.001, d=0.68$.). Dominance smiles also signaled more superiority $(M=64.2, S E=2.33)$ than positive feelings and reassurance $(M=58.2, S E=2.28), t(59)=3.43, p=0.001$, $d=0.44$. However, in this sample, affiliation smiles did not signal significantly more reassurance than positive feelings and superiority, $t(59)=0.35, p=0.73, d=0.05$.

The interaction between face presentation (visible, masked), smile type (reward, affiliation, dominance), and rating scale (target, non-target), $F(2,118)=4.79, p=0.01$, $\eta_{\mathrm{p}}{ }^{2}=0.075$, was significant. As tests reported in the caption (Fig. 4) indicate, we found support for the hypothesis according to which masks impair perception of the target signal in all smile types. However, the non-target signals were not perceived more in masked versus fully visible faces.

These findings were not moderated by political attitudes or gender of the participant (see S1 Appendix, Appendix 2). Further statistical analyses for Study 2 are also described in S1 Appendix, Appendix 2).

\section{Discussion}

If the eyes are the window to the soul, then masking faces during a pandemic should have no measurable consequences for the ability to perceive emotion in facial expressions accurately. Despite some evidence that information from the eyes is sufficient for the categorization of mental states (BaronCohen et al., 1997, 2001; Schmidtmann et al., 2020), informal testimony, however, suggests that the eyes alone are not enough: Individuals in service jobs regularly report that there have been frequent miscommunications about desires and concerns of customers due to the disruptive effects of masks
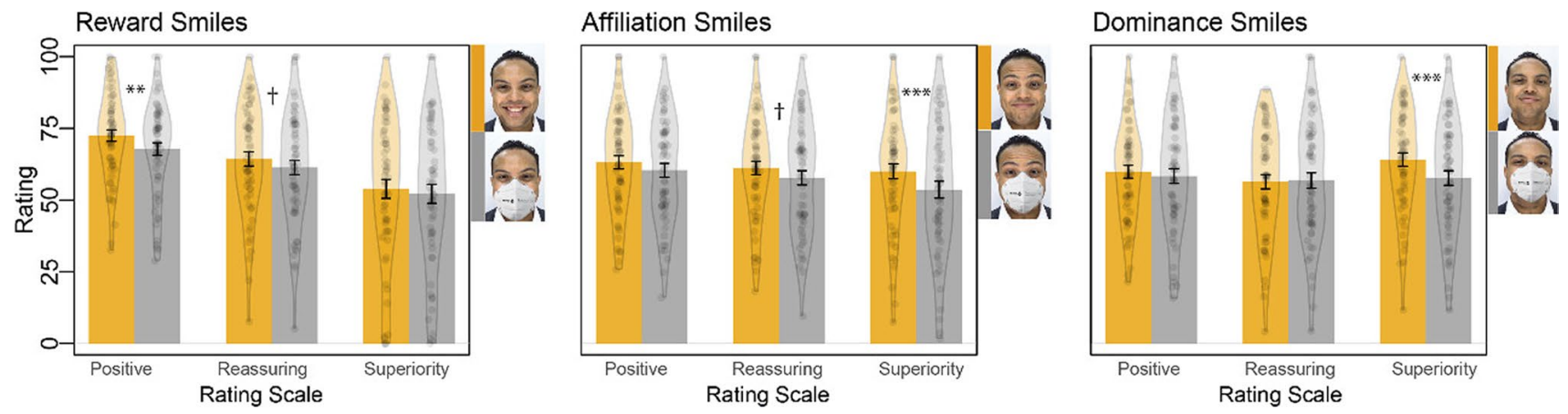

Fig. 7 Social signals of masked and visible smiles. The graphs illustrate levels of social signals communicated in videos of visible and masked faces expressing reward, affiliation, and dominance smiles. For reward smiles, masks reduced both the target signal (positive feeling; $p=.003$ ) and non-target signals $(p=.046)$, however, a marginally significant $(p=.072)$ between face presentation and rating scale interaction indicated that the effect was stronger for the target signal ratings than non-target signal ratings. For affiliation smiles, masks impaired perceptions of both the target (reassurance; $p=.06$ ) and non-target ratings $(p=.002)$, and the interaction was not significant $(p=.40)$. For dominance smiles, masks impaired perception of the target signal (superiority; $p<.001$ ) but not of non-target ratings $(p=.67)$. The interaction was significant $(p=.006) . \dagger p<.10,{ }^{*} p<.05$, $* * p<.01, * * * p<.001$ 
since the COVID-19 pandemic started (Bhattarai, 2020). The present findings lend empirical support to these reports.

In Study 1, we found that masked happy, disgust, anger, and surprise expressions were perceived as conveying significantly less happiness, disgust, anger, and surprise, respectively, than fully visible faces. The same expressions were also perceived as containing more of each of the other emotions. For example, happy expressions were seen as angrier and more disgusted which could have ramifications for both the perceiver and expressor. Findings for surprise varied from this pattern in one way such that masks obscured the perception of the amount of positive emotion (rated as happiness) conveyed by the surprise expression.

As expected, the effects of masks on the perception of emotion were larger for expressions that contain descriptively more signal from facial muscle contractions on the lower compared to upper part of the face. That is, much of the communicative signal from happy and disgust expressions is present in the contraction of the muscles on the part of the face specifically covered by protective masks (i.e., the lower part of the face) used during a pandemic. The extraction of information was somewhat less compromised for the expressions of anger and surprise, which involve more signal on the upper portion of the face.

In Study 2, the interpretation of dynamic smiles of reward, affiliation, and dominance that had been validated in previous work (Martin et al., 2018, 2021) was also compromised when the smiles were covered by face masks. This was observed in particular for reward and dominance smiles. When covered by masks, reward smiles were perceived as signaling less positive feeling and dominance smiles were perceived as signaling less superiority. Ratings of affiliation smiles as specifically communicating reassurance (the label we used for the signaling of non-threat) were only marginally lower in masked compared to visible conditions.

Previous research has found that affiliation smiles have their intended effects in social interaction (e.g., increasing trust) and are mentally represented as possessing a morphology that is distinct from smiles of reward and dominance (Rychlowska et al., 2017). Since the time that we selected the term "reassuring" to probe the perception of affiliation smiles for use in this study, other research in our laboratory found that the lay term that is most likely to be used for this smile is not "reassuring" but "fake" (Martin et al., 2021). This may be due to the fact that, except for "smirks" or "smug smiles" (i.e., dominance smiles in our terminology) which by definition do not signal that the smiler is feeling happiness, there is strong consensus in folk theory that "true" smiles always convey happiness. Thus, even though affiliation smiles may serve the function of communicating non-threat, they may be called "fake" colloquially because they are not, nor are they intended to be, signals of happiness. In future research, the impact of masked emotional expressions on subsequent social behavior and physiological responses would provide a stronger test of the hypothesis that the perception of emotion in facial expression is compromised by face masks (cf. Martin et al., 2018).

In the current study, masked stimuli were created by superimposing images of popular masks on existing expression stimuli. A limitation of this method is the reduction of some facial movement that may be seen when the expressor is wearing the mask. The face mask is fixed in size and position relative to the face which may limit the perception of lower face movement. In light of this limitation, the effect sizes discussed here, especially for emotions that predominantly utilize the lower part of the face, may overestimate those seen in natural settings. Further, the superimposition of masks on pre-existing dynamic facial expressions does impact the continuity of the stimuli. That is, despite the stimuli approximating naturally occurring masked expressions, they may be perceived as unnatural. Superimposing masks may draw attention to the mask over and beyond the amount seen during social interactions during the COVID-19 pandemic.

\section{Future Directions}

During the COVID-19 pandemic, people in all countries of the world have received recommendations for positive health behaviors, and the wearing of face masks is one such recommendation. This begs the question of whether the perception of emotion expressions and facial signals of other internal states is hindered by mask wearing across cultures. Recent research on culture and emotion suggests that any detrimental effects of mask wearing are likely not uniform across cultures.

Specifically, there are differences in how much emphasis cultures place on the explicit facial display of internal states of emotion (Matsumoto et al., 2008). Rychlowska and colleagues (Rychlowska et al., 2015) reported that people in countries of the world with ancestrally diverse populations (i.e., those in which historical migration patterns have produced a society composed of people from many different countries) tend to encourage the overt display of emotion on the face when emotion is felt internally. In contrast, people in less ancestrally diverse countries tend to encourage the suppression of explicit displays of emotion. The difference may in part be due to the fact that in places of high ancestral diversity there is relatively low consensus about which emotions are experienced in which contexts (Gelfand et al., 2011; Masuda et al., 2008). Facial expressions may thus provide information that context does not (Niedenthal et al., 2019). This reasoning suggests that there will be cultural differences in the effects of mask wearing such that masks are more detrimental to the processing of facial expression in societies of high compared to low ancestral diversity. Further, there is some evidence that 
there are cultural differences in gaze patterns during facial expression recognition. Specifically, the importance of the mouth in the recognition of facial expressions may not be universal. Jack et al. (2012) showed that internal representations of emotion focus more on the eyes for people from East Asian cultures compared to the eyes and the mouth for people from Western cultures. Assessment of such differences will necessitate the study of facial expression in context rather than in isolation as in the present studies.

Even within cultures there are likely to be changes in any effects of mask wearing over the period of a pandemic. Characteristics of both perceivers and mask wearers could plausibly contribute to these changes. Over time, perceivers may come to make more eye contact with mask wearers. Eye contact can trigger a more accurate simulation of the perceived expression and its corresponding state in the perceiver for use in interpreting the emotion signal (Wood et al., 2016). Mask wearers may begin to intentionally and ultimately automatically increase signals to their underlying emotions in the uncovered (upper) versus covered (lower) parts of their faces. Mask wearers may also begin to increase the expression of their emotions in modalities that are not occluded by masks such as the voice (Laukka, 2005). In addition, they may begin to use conventional gestures to complete or augment emotion signals from other expressive modalities (Wood et al., 2019). Given the decreased perception of happiness in a mask-covered smile observed in the present research, increasing other signals of positive emotion will be essential for everyday life.

\section{Conclusion}

The findings reported here, in combination with other recent research (Carbon \& Serrano, 2021; Carbon, 2020; Marini et al., 2021) support the recommendation that people increase the communication of emotion through modalities that are not compromised by face masks such as verbal methods of communicating emotion and the use of conventional gesture. This recommendation may prove to be especially useful for emotions that utilize the lower (happiness, disgust) as opposed to upper part of the face (surprise, anger). Our finding, that non-target emotions were rated higher for masked faces, further supports this recommendation in cases where other cues may be limited. This includes ambiguous social situations which may support multiple interpretations of a masked expression.

Acknowledgements The authors thank Ann Shaffer for her expert editing and Sari Judge for her communication expertise. Markus Brauer provided statistical consulting and Carolin Showers gave detailed feedback on a version of the manuscript.

\section{Additional Information}

Funding Information Dasha A. Yermol received a University of Wisconsin-Madison Hilldale Fellowship for her efforts on this project.

Data Availabity The materials and data used in this research are publicly available at https://osf.io/rbhxd/?view_only=1f01c0205c884f5 4b7472fb3a617c15b.

Ethics Approval The study was approved by the University of Wisconsin, Madison IRB.

Conflicts of Interest The authors declare no competing interests.

Informed Consent All participants consented to the research before beginning the study.

Supplementary Information The online version contains supplementary material available at https://doi.org/10.1007/s42761-021-00097-z.

Author Contribution A.T.L., F.Z., D.A.Y., C.A.T., and P.M.N designed research; A.T.L. and D.A.Y. performed research; F.Z. and C.A.T. analyzed data; and A.T.L., F.Z., and P.M.N. wrote the paper.

\section{References}

Ambadar, Z., Schooler, J. W., \& Cohn, J. F. (2005). Deciphering the enigmatic face: The importance of facial dynamics in interpreting subtle facial expressions. Psychological Science, 16(5), 403-410. https://doi.org/10.1111/j.0956-7976.2005.01548.x

Back, E., Jordan, T. R., \& Thomas, S. M. (2009). The recognition of mental states from dynamic and static facial expressions. Visual Cognition, 17(8), 1271-1286. https://doi.org/10.1080/13506 280802479998

Baron-Cohen, S., Wheelwright, S., \& Jolliffe, T. (1997). Is there a "Language of the Eyes"? Evidence from normal adults, and adults with autism or Asperger syndrome. Visual Cognition, 4, 311-331. https://doi.org/10.1080/713756761

Baron-Cohen, S., Wheelwright, S., Hill, J., Raste, Y., \& Plumn, I. (2001). The "Reading the Mind in the Eyes" Test Revised Version: Study with Normal Adults, and Adults with Aspergers Syndrome or High-functioning Autism. Journal of Child Psychology and Psychiatry and Allied Discipline, 42(2), 241-251. https://doi.org/ 10.1017/S0021963001006643

Bhattarai, A. (2020) Cranky customers, masked smiles redefine workplace culture: 'Feels like we're strangers'. Available at: https:// www.washingtonpost.com/road-to-recovery/2020/09/23/masksworkplace-culture-pandemic. Accessed Nov 232020

Blais, C., Roy, C., Fiset, D., Arguin, M., \& Gosselin, F. (2012). The eyes are not the window to basic emotions. Neuropsychologia, 50(12), 2830-2838. https://doi.org/10.1016/j.neuropsychologia. 2012.08.010

Calvo, M. G., \& Nummenmaa, L. (2008). Detection of emotional faces: Salient physical features guide effective visual search. Journal of Experimental Psychology: General, 137, 471-494. https://doi.org/ $10.1037 / \mathrm{a} 0012771$

Carbon, C. C. (2020). Wearing face masks strongly confuses counterparts in reading emotions. Frontiers in Psychology, 11, 566886. https://doi.org/10.3389/fpsyg.2020.566886

Carbon, C. C., \& Serrano, M. (2021). The impact of face masks on the emotional reading abilities of children - a lesson from a joint 
school - university project. I-perception. 12(4). https://doi.org/10. $1177 / 20416695211038265$

Cattaneo, L., \& Pavesi, G. (2014). The facial motor system. Neuroscience and Biobehavioral Review, 38, 135-159. https://doi.org/10. 1016/j.neubiorev.2013.11.002

Crivelli, C., \& Fridlund, A. J. (2014). Facial displays are tools for social influence. Trends in Cognitive Science, 22(5), 388-399. https:// doi.org/10.1016/j.tics.2018.02.006

Ekman, P., Friesen, W. V., \& Tomkins, S. S. (1971). Facial affect scoring technique: A first validity study. Semiotica, 3, 37-58.

Ekman, P., Friesen, W. V., Hagar, J. (2002) C Facial Action Coding System (pp. 15-464). Research Nexus division of Network Information Research Corporation.

Fischer, A. H., Gillebaart, M., Rotteveel, M., \& Becker, D. (2012). Veiled Emotions. Social Psychological and Personality Science, 3(3), 266-273. https://doi.org/10.1177/1948550611418534

Gelfand, M. J., Raver, R. L., Nishii, L. M., Lun, J., Lim, B. C., Duan, L., ... Yamaguchi, S. (2011). Differences between tight and loose cultures: A 33-nation study. Science, 332, 1100-1104. https://doi. org/10.1126/science.1197754

Hassin, R. R., Aviezer, H., \& Bentin, S. (2013). Inherently ambiguous: Facial expressions of emotions, in context. Emotion Review, 5(1), 60-65. https://doi.org/10.1177/1754073912451331

Hastings, M. E., Tangney, J. P., \& Stuewig, J. (2008). Psychopathy and identification of facial expressions of emotion. Personality and Individual Differences, 44(7), 1474-1483. https://doi.org/10. 1016/j.paid.2008.01.004

Jack, R. E., Caldara, R., \& Schyns, P. G. (2012). Internal representations reveal cultural diversity in expectations of facial expressions of emotion. Journal of Experimental Psychology: General, 141(1), 19. https://doi.org/10.1073/pnas.1200155109

Jack, R. E., \& Schyns, P. G. (2015). The human face as a dynamic tool for social communication. Current Biology, 25(14), R621-R634. https://doi.org/10.1016/j.cub.2015.05.052

Kamachi, M., Bruce, V., Mukaida, S., Gyoba, J., Yoshikawa, S., \& Akamatsu, S. (2013). Dynamic properties influence the perception of facial expressions. Perception, 42(11), 1266-1278. https://doi. org/10.1068/p3131n

Keltner, D., Ekman, P., Gonzaga, G. C., \& Beer, J. Facial expression of emotion. In Davidson, R. J., Scherer, K. R., \& Goldsmith, H. H. (2003) (Ed.), Series in affective science. Handbook of affective sciences (pp. 415-432). Oxford University Press. https://doi.org/ 10.1007/s10919-019-00293-3

Kret, M. E., Prochazkova, E., Sterck, E. H., \& Clay, Z. (2020). Emotional expressions in human and non-human great apes. Neuroscience and Biobehavioral Review, 115, 378-395. https://doi.org/10. 1016/j.neubiorev.2020.01.027

Laukka, P. (2005). Categorical perception of vocal emotion expressions. Emotion, 5(3), 277-295. https://doi.org/10.1037/15283542.5.3.277

Maringer, M., Krumhuber, E. G., Fischer, A. H., \& Niedenthal, P. M. (2011). Beyond smile dynamics: Mimicry and beliefs in judgments of smiles. Emotion, 11(1), 181-187. https://doi.org/10. 1037/a0022596

Marini, M., Ansani, A., Paglieri, F., Caruana, F., \& Viola, M. (2021). The impact of facemasks on emotion recognition, trust attribution and re-identification. Scientific Report, 11, 5577. https://doi.org/ 10.1038/s41598-021-84806-5

Marsh, A. A., \& Blair, R. J. R. (2008). Deficits in facial affect recognition among antisocial populations: A meta-analysis. Neuroscience and Biobehavioral Review, 32(3), 454-465. https://doi.org/ 10.1016/j.neubiorev.2007.08.003
Martin, J., Rychlowska, M., Wood, A., \& Niedenthal, P. M. (2017). Smiles as multipurpose social signals. Trends in Cognitive Science, 21(11), 864-877. https://doi.org/10.1016/j.tics.2017.08.007

Martin, J. D., Abercrombie, H. C., Gilboa-Schechtman, E., \& Niedenthal, P. M. (2018). Functionally distinct smiles elicit different physiological responses in an evaluative context. Scientific Report, 8(1), 3558. https://doi.org/10.1038/s41598-018-21536-1

Martin, J. D., Wood, A., Cox, W. T. L., Sievert, S., Nowak, R., Gilboa-Schechtman, E., ... Niedenthal, P. M. (2021). Evidence for Distinct Facial Signals of Reward, Affiliation, and Dominance from Both Perception and Production Tasks. Affective Science, 2, 14-30. https://doi.org/10.1007/s42761-020-00024-8

Masuda, T., Ellsworth, P. C., Mesquita, B., Leu, J., Tanida, S., \& Van de Veerdonk, E. (2008). Placing the face in context: Cultural differences in the perception of facial emotion. Journal of Personality and Social Psychology, 94(3), 365-381. https://doi.org/10. 1037/0022-3514.94.3.365

Matsumoto, D., Yoo, S. H., \& Fontaine, J. (2008). Mapping expressive differences around the world: The relationship between emotional display rules and individualism versus collectivism. Journal of Cross-Cultural Psychology, 39(1), 55-74. https://doi.org/10.1177/ 0022022107311854

Niedenthal, P. M., Mermillod, M., Maringer, M., \& Hess, U. (2010) The Simulation of Smiles (SIMS) model: Embodied simulation and the meaning of facial expression. Behavioral and Brain Science, 33, 417-480. https://doi.org/10.1017/S0140525X1 0000865

Niedenthal, P. M., \& Brauer, M. (2011). Social functionality of human emotions. In S.T. Fiske (Ed.). Annual Review of Psychology, 62, 259-285. https://doi.org/10.1146/annurev.psych.121208.131605

Niedenthal, P. M., Rychlowska, M., Zhao, F., \& Wood, A. (2019). Historical Migration Patterns Shape Contemporary Cultures of Emotion. Perspectives on Psychological Science, 14(4), 560-573. https://doi.org/10.1177/1745691619849591

Nusseck, M., Cunningham, D. W., Wallraven, C., \& Bülthoff, H. H. (2008). The contribution of different facial regions to the recognition of conversational expressions. Journal of Vision., 8(8), 1. https://doi.org/10.1167/8.8.1

Pew Research Center. (2020). Republicans, Democrats Move Even Further Apart in Coronavirus Concerns. Available at: https:// www.pewresearch.org/politics/2020/06/25/republicans-democ rats-move-even-further-apart-in-coronavirus-concerns. Accessed 13 Nov 2020

Rychlowska, M., Miyamoto, Y., Matsumoto, D., Hess, U., Gilboa-Schechtman, E., Kamble, S., Muluk, H., Masuda, T., \& Niedenthal, P. M. (2015). Heterogeneity of long-history migration explains cultural differences in reports of emotional expressivity and the functions of smiles. Proceedings of the National Academy of Science United States of America, 112(19), E2429-E2436. https://doi.org/10.1073/pnas.1413661112

Rychlowska, M., Jack, R. E., Garrod, O., Schyns, P. G., Martin, J. D., \& Niedenthal, P. M. (2017). Functional Smiles: Tools for Love, Sympathy, and War. Psychological Science, 28(9), 1259-1270. https://doi.org/10.1177/0956797617706082

Saumure, C., Plouffe-Demers, M.-P., Estéphan, A., Fiset, D., \& Blais, C. (2018). The use of visual information in the recognition of posed and spontaneous facial expressions. Journal of Vision, 18(9), 21-21. https://doi.org/10.1167/18.9.21

Schmidtmann, G., Logan, A. J., Carbon, C.-C., Loong, J. T., \& Gold, I. (2020). In the Blink of an Eye: Reading Mental States From Briefly Presented Eye Regions. i-Perception (London), 11(5). https://doi.org/10.1177/2041669520961116 
Smith, M. L., Cottrell, G., Gosselin, F., \& Schyns, P. G. (2005). Transmitting and decoding facial expressions of emotions. Psychological Science, 16, 184-189. https://doi.org/10.1111/j.0956-7976. 2005.00801.x

Thompson, A. E., \& Voyer, D. (2014). Sex differences in the ability to recognize non-verbal displays of emotion: A meta-analysis. Cognition and Emotion, 28(7), 1164-1195. https://doi.org/10.1080/ 02699931.2013.875889

Wood, A., Rychlowska, M., Korb, S., \& Niedenthal, P. M. (2016). Fashioning the face: Sensorimotor simulation contributes to facial expression recognition. Trends in Cognitive Science, 20(3), 227240. https://doi.org/10.1016/j.tics.2015.12.010

Wood, A., Martin, J. D., Alibali, M. W., \& Niedenthal, P. M. (2019). A sad thumbs up: Incongruent gestures and disrupted sensorimotor activity both slow processing of facial expressions. Cognition and Emotion, 33(6), 1196-1209. https://doi.org/10.1080/02699 931.2018.1545634 